\title{
QUANTUM RENORMALIZATION FOR THE ANISOTROPIC HEISENBERG MODEL*
}

\author{
R. DEKEYSER, M. REYNAERT and M.H. LEE $\dagger$ \\ Instituut voor Teoretische Fysika, Katholieke Universiteit Leuven, Celestijnenlaan 200 D, B-3030 Heverlee, Belgium
}

\begin{abstract}
It is shown that the quantum renormalization method may be applied to the two-dimensional spin $\frac{1}{2}$ anisotropic Heisenberg model. An appropriate set of transformations is chosen in such a way that the symmetry properties of the hamiltonian are not disturbed by the renormalization. The fixed points and the flow diagram are determined.
\end{abstract}

Recently, attempts have been made to extend the renormalization group techniques of Niemeijer and van Leeuwen [1] to quantum models $[2,3]$. If the original hamiltonian $\mathscr{H}\left(\left\{\boldsymbol{S}_{j}\right\}\right)$ is expressed in terms of the spin vectors $S_{j}$ at the lattice sites $j$, the essential steps in this method are:

(a) The usual choice of cells, numbered by an index $J$.

(b) A unitary transformation, within each cell, from the base vectors in spin space $\left|\left\{S_{j}^{z}\right\}\right\rangle$ to a new base $\left|S_{J}^{z}, \tau_{J}\right\rangle$, where $S_{J}^{z}$ will be the $z$-component of the cell spin and $\tau_{J}$ is a dummy variable.

(c) The evaluation of the renormalized cell spin hamiltonian $\mathscr{H}^{\prime}\left(\left\{\boldsymbol{S}_{J}\right\}\right)$ by taking the partial traces over the $\tau_{J}$ :

$$
\left\langle\left\{S_{J}^{z}\right\}\left|\mathrm{e}^{\mathscr{K}}\right|\left\{S_{J}^{z^{\prime}}\right\}\right\rangle \equiv \sum_{\left\{\tau_{J}\right\}}\left\langle\left\{S_{J}^{z}, \tau_{J}\right\}\left|\mathrm{e}^{\mathscr{K}}\right|\left\{S_{J}^{z^{\prime}}, \tau_{J}\right\}\right\rangle
$$

or

$$
\left\langle\left\{S_{J}^{z}\right\}\left|\mathrm{e}^{\mathscr{K}}\right|\left\{S_{J}^{z^{\prime}}\right\}\right\rangle \equiv \sum_{k} p_{k} \sum_{\left\{\tau_{J}\right\}}\left\langle\left\{S_{J}^{z}, \tau_{J}\right\} ; k\left|\mathrm{e}^{\mathscr{K}}\right|\left\{S_{J}^{z^{\prime}}, \tau_{J}\right\} ; k\right\rangle
$$

if one wishes to take an average (with normalized weight factors $p_{k}$ ) over several choices of unitary transformations from step $b$.

One may need to use the more complicated formula (2) in order to preserve, through the renormalization, some symmetry properties of the hamiltonian. The simple formula (1) disturbs the isotropy of the Heisenberg hamiltonian, since it allows the $z$-axis to play a special role (see ref. 2). One thus needs similar transformations where the $z$-axis is replaced by the $x$ -

\footnotetext{
* This research has been partially supported by a NATO Research Grant, No. 1024.

$\dagger$ Permanent address: University of Georgia, Athens, USA,
}

and $y$-axes. The general procedure is as follows.

Suppose the hamiltonian is invariant under a group $\mathrm{G}$, whose elements $k$ are unitarily represented in the space of the $j$ th spin by $U(k, j)$. The renormalized hamiltonian will then preserve the symmetry of the group $G$ if we use formula (2) with all basic sets of the form

$$
\left|S_{J}^{z}, \tau_{J} ; k\right\rangle \equiv U^{+}(k, J) \prod_{j \in J} U(k, j)\left|S_{J}^{z}, \tau_{J}\right\rangle
$$

In approximate calculations it is not necessary in eq. (2) to average over the whole group, but an averaging over an appropriate subgroup can do the same job, as will be illustrated in what follows.

We have applied this method to the anisotropic spin $\frac{1}{2}$ Heisenberg hamiltonian

$$
\mathscr{H}=\sum_{\langle i j\rangle}\left(K_{1}\left(S_{i}^{+} S_{j}^{-}+S_{j}^{+} S_{i}^{-}\right)+2 K_{2} S_{i}^{z} S_{j}^{z}\right)
$$

where the sum runs over all nearest-neighbour pairs on a triangular lattice. We have chosen the triangular cells as in ref. 1; and we may write

$$
\mathscr{H}=\mathscr{H}_{0}+V
$$

where $\mathscr{H}_{0}$ contains all intracell interactions.

Starting with the simple set of new base vectors

$$
\begin{array}{ll}
|+, 1\rangle=|++-\rangle & |-, 1\rangle=|--+\rangle \\
|+, 2\rangle=|+-+\rangle & |-, 2\rangle=|-+-\rangle \\
|+, 3\rangle=|-++\rangle & |-, 3\rangle=|+--\rangle \\
|+, 4\rangle=|+++\rangle & |-, 4\rangle=|---\rangle
\end{array}
$$

we have obtained new sets by the procedure (3), using the subgroup of $\mathrm{O}_{3}$, generated by the rotations of $90^{\circ}$ around the $x-, y$ - and $z$-axes and 
by the inversion. In order to obtain the renormalized hamiltonian, we apply the BakerCampbell-Hausdorff formula and the cumulant expansion as explained in refs. 2 and 3. Anisotropic Heisenberg interactions between next and third nearest neighbours are generated by the same procedure, and their interaction strengths are labeled $K_{3}-K_{6}$. The resulting renormalization equations,

$K_{j}^{\prime}=f_{j}\left(K_{1}, \ldots, K_{6}\right)$,

have been evaluated up to second order in $K_{1}$ and $K_{2}$ (this is to first order in $K_{3}-K_{6}$ ). These equations now preserve the isotropy since, e.g.

$f_{1}(a, a, b, b, c, c)=f_{2}(a, a, b, b, c, c)$.

Eqs. (7) exhibit fixed points at $K_{j}=0$ and at infinity, and also at three non-trivial fixed points:

(a) a Heisenberg fixed point at $K_{1}=K_{2}=$ $1.427, K_{3}=K_{4}=0.360, K_{5}=K_{6}=0.0095$;

(b) an Ising fixed point at $K_{1}=K_{3}=K_{5}=0$, $K_{2}=0.699, K_{4}=0.135, K_{6}=0.045$; and

(c) an $X Y$ fixed point at $K_{1}=0.739, K_{2}=$ $0.032, K_{3}=0.137, K_{4}=-0.00002, K_{5}=0.043$, $K_{6}=-0.00003$.

The Ising and $X Y$ fixed points have the correct orders of magnitude [note in the definition (4) the difference from the usual Ising expansion parameter by a factor 2]. The Heisenberg fixed point is unreliable since $K_{1}$ is too large to allow us to truncate the cumulant expansion after the second order. It may well be that in a higher

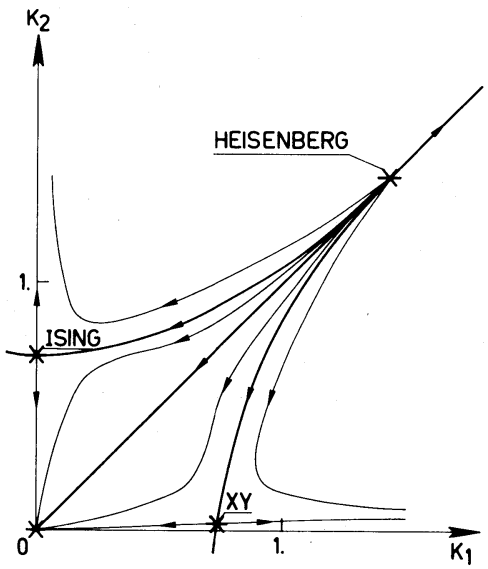

Fig. 1. Fixed points and flow diagram for the renormalization equations of the anisotropic Heisenberg model (projection on the $\left(K_{1}, K_{2}\right)$-plane).

order calculation this fixed point disappears, or it shifts to infinity $\left(T_{\mathrm{c}}=0\right)$. Linearization around this point yields an anisotropy crossover exponent $\phi \simeq 6.8$ (in $1 / n$ expansion [4]) it is infinity at $d=2$ ).

Although the numerical results may be further improved, the resulting flow diagram between the fixed points (see fig. 1) clearly shows the qualitative features that one believes will persist for $d=3$.

\section{References}

[1] Th. Niemeijer and J.M.J. van Leeuwen, Phys. Rev. Lett. 31 (1973) 1411, and Physica 71 (1974) 17.

[2] J. Rogiers and R. Dekeyser, Phys. Rev. B13 (1976) 4886.

[3] D.D. Betts and M. Plischke, preprint.

[4] S. Hikami and R. Abe, Progr. Theor. Phys. 52 (1974) 369. 